\title{
Predictive motor activation: Modulated by expectancy or predictability?
}

Tommaso Ghilardia, Marlene Meyer ${ }^{\mathrm{a}}$, Sabine Hunnius ${ }^{\mathrm{a}}$

a) Donders Institute for Brain, Cognition and Behaviour, Radboud University Nijmegen Thomas van Aquinostraat 4, 6525GD Nijmegen, The Netherlands

Corresponding author

Tommaso Ghilardi, Tommaso.ghilardi@donders.ru.nl

\section{Abstract}

Predicting actions is a fundamental ability that helps us to comprehend what is happening in our environment and to interact with others. The motor system was previously identified as source of action predictions. Yet, which aspect of the statistical likelihood of upcoming actions the motor system is sensitive to remains an open question. This EEG study investigated how regularities in observed actions are reflected in the motor system and utilized to predict upcoming actions. Prior to measuring EEG, participants watched videos of action sequences with different transitional probabilities. After training, participants' brain activity over motor areas was measured using EEG while watching videos of action sequences with the same statistical structure. Focusing on the mu and beta frequency bands we tested whether activity of the motor system reflects the statistical likelihood of upcoming actions. We also explored two distinct aspects of the statistical structure that capture different prediction processes, expectancy and predictability. Expectancy describes participants' expectation of the most likely action, whereas predictability represents all possible actions and their relative probabilities. Results revealed that mu and beta oscillations play different roles during action prediction. While the mu rhythm reflected anticipatory activity without any link to the statistical structure, the beta rhythm was related to the expectancy of an action. Our findings support theories proposing that the motor system underlies action prediction, and they extend such theories by showing that multiple forms of statistical information are extracted when observing action sequences. This information is integrated in the prediction generated by the neural motor system of which action is going to happen next. 


\section{Research Highlights}

- Adults learn how action sequences unfold through visual statistical learning and generate expectations about future actions.

- Knowledge about action sequences is fed into the neural motor system to predict upcoming actions.

- Suppression of mu and beta rhythms reflects different statistical information during action prediction.

- Mu rhythm reflects action prediction independently of the statistical structure

- Beta rhythm reflects prediction of the most probable upcoming actions

\section{Keywords}

Action prediction; Statistical learning; Motor cortex; Transitional probabilities; Mu rhythm; Beta rhythm; 


\section{Introduction}

Action prediction is a fundamental skill that allows us to anticipate how an action we observe will likely unfold (Flanagan \& Johansson, 2003). For instance, when we see someone reaching for a cookie, we readily anticipate they will bring the cookie to their mouth to eat it. Yet, in everyday life, predicting actions is often not that simple. Only few actions are linked to each other in a deterministic fashion. Rather, in most cases, one action can precede a variety of different subsequent actions. Re-examining the cookie example, it is easy to imagine that grabbing a cookie could be followed by various actions: the cookie could be eaten, it could be soaked in milk, it could be thrown away or broken in half to share it with someone. Thus, to successfully predict actions we need to account for multiple possibilities and their different probabilities. In real life multiple sources of information can help us in predicting which action will be performed next: the kinematics of the action (Rutkowska et al., 2021; Stadler et al., 2012; Stapel et al., 2012), the affordance of the manipulated object (Bach et al., 2014) and the context of the situation (Hudson et al., 2016; Stapel et al., 2012). In this study, we will focus on the probabilities linking different actions, a crucial aspect for determining which action to predict next.

Predictive processing theories propose that to identify the most probable action we integrate the current sensory information with prior knowledge (Kahl \& Kopp, 2018; Kilner et al., 2007; Ondobaka et al., 2015; Wolpert et al., 2003). For instance, we might have grown up with a sibling who loves soaking cookies in milk and only rarely shares them (prior knowledge). According to such probabilistic accounts, we integrate our current observation (i.e., someone reaching for a cookie) with our prior knowledge to predict the most probable subsequent action.

Learning about associations between events through repeated experience of their cooccurrence or succession is called statistical learning (Fiser \& Aslin, 2002). In the context of action observation, recent studies demonstrated that adults are able to extract statistical regularities from continuous streams of human action (Ahlheim et al., 2014; Baldwin et al., 2008) and use the acquired knowledge to make predictive eye movements to the location of an action before it occurs (Monroy et al., 2018). The prediction of an upcoming action has been traced back to the motor cortex (Debnath et al., 2019; Elsner et al., 2013; Kilner et al., 2004), and the same areas that are active while performing an action are thus involved in predicting others' actions (Flanagan \& Johansson, 2003; Kilner, 2011; Stapel et al., 2010). In electroencephalographic (EEG) measurements, power in the mu $(9-11 \mathrm{~Hz})$ and beta $(12-30 \mathrm{~Hz})$ frequency ranges over motor areas has been shown to be modulated during action execution, action observation, and during prediction of actions (Caetano et al., 2007; Muthukumaraswamy et al., 2006; Oberman et al., 2005). Converging evidence suggests that activation in the motor areas during action observation reflects the prediction of an upcoming action in the motor system (Elsner et al., 2013; Stapel et al., 2016), and, recently, a study demonstrated that such motor predictions can be based on statistical regularities learned through observation (Monroy et al., 2019). However, Monroy and colleagues (2019) 
compared action sequences that were $100 \%$ predictable (i.e., deterministic) with random actions, whereas action prediction in everyday life requires us to extract multiple different transitional probabilities from action sequences and use this information to predict what is going to happen. There are first indications that the probability of upcoming actions is reflected in the activation of the observer's neural motor system (Braukmann et al., 2017; Monroy et al., 2019; Tzagarakis et al., 2010): Using different methodologies, these studies report that activity of the sensorimotor areas after perceiving an action is modulated by the probability of the following one.

While the results of these studies show that the motor cortex is involved in predicting upcoming actions, they do not explain how the most likely upcoming action is determined. The expectation of what is going to happen after a certain action is observed, can be expressed by two different measures that are based on the number of possible next actions and their probability: An action's expectancy is determined by its probability and reflects the participant's expectation of what action is most likely performed next. On the other hand, the number and probability of all possible actions determines the action's predictability. This measure reflects the distribution of how many alternative actions there are and how likely each of them is, and as such the predictability of an action can vary independently of its absolute probability. Using the example previously presented, the expectancy that a cookie is brought to the mouth after grasping it depends on the number of times this specific action has been observed after grasping the biscuit. Predictability, on the other hand, is not determined by the specific action but by the number of all possible actions (the cookie could be eaten, it could be soaked in milk, it could be thrown away or broken in half to share it with someone) and their probabilities. In the context of action prediction, these two aspects describe different ways of predicting upcoming actions. The activity in our motor system might reflect the expectancy of the most likely next action (i.e., the action with highest probability), thereby ignoring the different options. Alternatively, our motor system might represent all possible actions and their relative probabilities. In an fMRI study, (Ahlheim et al., 2014)) explored the brain activity related to two similar statistical properties in an action sequence. Results demonstrated that the two statistical properties could be distinguished with regard to their neural correlates. This study provides the first evidence that different statistical properties can be extracted from an action sequence, but it leaves a crucial question unanswered. Which statistical property we rely on when predicting upcoming actions?

The current study tested whether predictive motor system activation reflects different transitional probabilities between actions in a sequence and if so, which statistical property of the environment (i.e. expectancy or predictability) best explains mu and beta rhythms. We used a similar paradigm as Monroy and colleagues (Monroy et al., 2019) with the crucial manipulation of parametrically varying the transitional probabilities between actions. In our study, human adults received visual experience with a sequence of actions for 34 minutes distributed over three days at home. They observed videos of action pairs containing four different levels of transitional probability (25\%, 50\%, 75\%, 100\%). Following the training, participants took part in an EEG experiment in which they observed two novel action sequences featuring the same statistical structure of actions as in the training phase, while their neural motor activity was recorded. To assess whether the motor system represents the 
transitional probabilities between actions in a sequence, we assessed the power in the mu $(9-11 \mathrm{~Hz})$ and beta $(12-30 \mathrm{~Hz})$ frequency band over motor cortices in the time window between two actions. We hypothesized that power in mu and beta frequency bands would be modulated parametrically by transitional probability, resulting in the highest activation for actions that are $100 \%$ probable and lowest activation for actions that are only $25 \%$ probable. We had no specific hypotheses on which of the two measures of the statistical structure better represented the activity over the motor area.

\section{Methods}

\subsection{Participants}

In total, 33 right-handed participants took part in this study of which 28 were included in the EEG data analysis (19 females) and five were excluded because they failed to complete the training. They were recruited from a pool of 18- to 55-year-old volunteers. Participants were all healthy adults with normal or corrected to normal vision and hearing. The university's Social Sciences Faculty's ethics committee approved the study (approval number ECSW20160905-396). Participants signed informed consent in accordance with the Declaration of Helsinki and received course credits or a monetary compensation for their participation.

\subsection{Stimuli and materials}

The stimulus materials included the same action videos as in the studies by (Monroy et al., 2018, 2019) with the only modifications being the statistical structure (i.e. transitional probabilities between actions). Five video sequences were created, three training videos and two test videos, with the same duration (11.27 minutes). Videos featured action sequences that involved a box-shaped toy which afforded six unique object-directed actions (see Figure 1). Action sequences were formed by a pair of actions, a cue (first) action and a paired (second) action, that were linked by different levels of probability. Figure1 illustrates the presentation of an action pair. Each trial started with the 1-second presentation of a fixation cross followed by a 1-second still frame of the toy-box. The two actions, each with a duration of 2 seconds, were performed consecutively. The two actions were interleaved with a still frame of the toy-box presented for 1 second, constituting the predictive time window of interest. Each stimulus video featured 32 deterministic pairs, 64 pairs with a transitional probability of $75 \%, 64$ pairs with a transitional probability of $50 \%$, and 32 pairs with a transitional probability of $25 \%$. Thus, participants were presented with 192 actions for each video for a total of 576 actions during the three days of the learning phase. All cue and paired actions were presented an identical number of times (i.e., 16) to ensure that participants received equal exposure to all six actions. Videos were divided into four blocks. Between each block videos of moving shapes were presented for four seconds as attention getters. In each block, the orientation of the toy-box relative to the camera was rotated to ensure that 
participants could not simply learn the physical location of the actions on the screen. In addition, as in Monroy et al. (Monroy et al., 2019), we imposed the following constraints: no pair could occur more than two times consecutively and pairs could not consist of the same action twice. Thus, all stimulus features were identical to Monroy et al. (Monroy et al., 2019) except for the variation in transitional probabilities that we added.

\section{Example of deterministic and high probability trials}

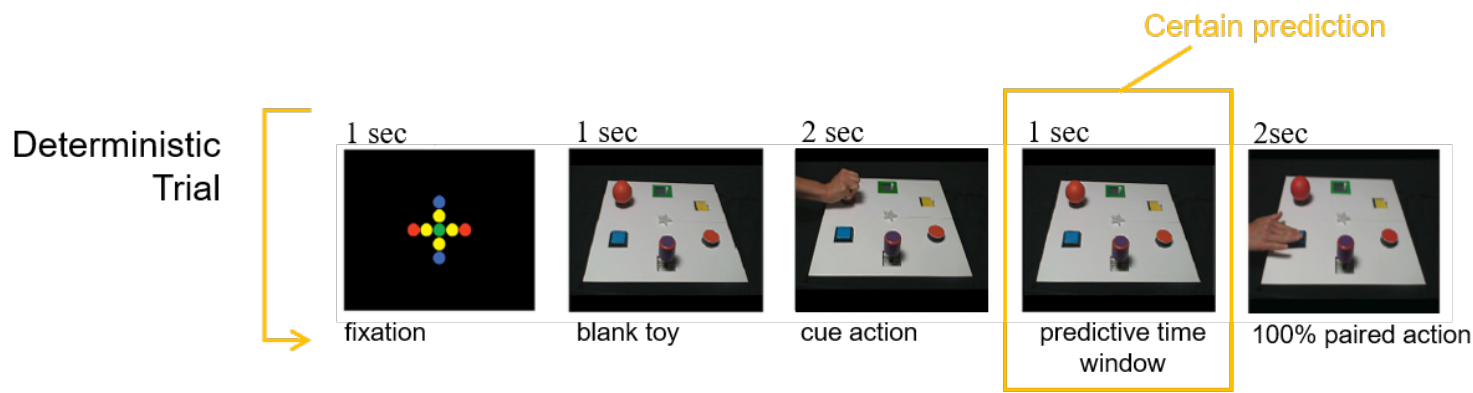

High probability

Trial

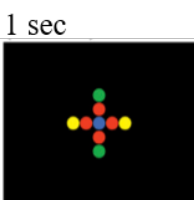

fixation

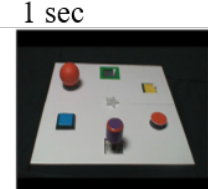

blank toy

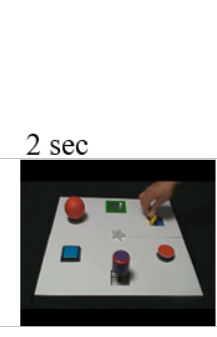

cue action

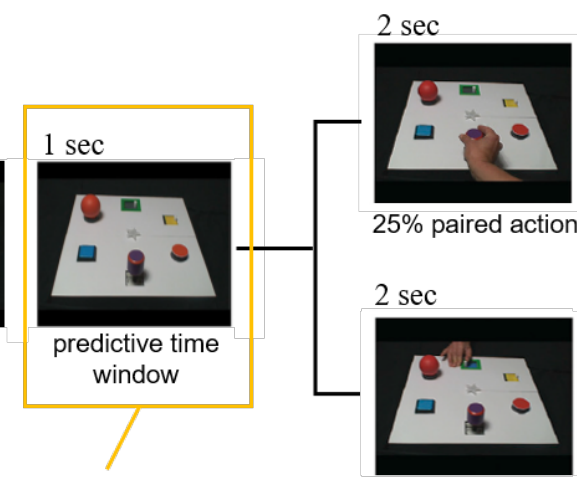

$75 \%$ paired action

Figure 1. Example frames from video stimuli illustrating two trials. Trials began with a static fixation cross, followed by four successive clips (7s total). EEG markers were time-locked to the onset of each clip. Yellow boxes indicate that time window used for comparison in the EEG analysis: conditions were perceptually identical (a 1s still frame with no action occurring), but in the Deterministic condition, participants could make a specific prediction about the subsequent action based on statistical likelihood, whereas in the High probability condition they could make predictions about two different outcomes with different probabilities. The fixation cross was used as a baseline in the analysis. 


\subsection{Probabilities}

To be able to dissociate the impact of expectancy and predictability of an action prediction, we created action sequences according to a transitional matrix. The different levels of transitional probability are shown in Figure 2: deterministic (100\%), high probability $(75 \%)$, medium probability (50\%), and low probability (25\%). Deterministic pairs consisted of two actions that always occurred together (i.e., $100 \%$ predictable). In the first high probability pair, a cue action was followed $75 \%$ of the time by a paired action and $25 \%$ of the time by another action (for instance, 'slide' was followed $75 \%$ of the time by 'tilt' and $25 \%$ of the time by 'open'). In the second high probability pair, the cue action was followed by three actions: one with $75 \%$ probability and the other two with $12.5 \%$. The medium probability pairs consisted of two cue actions: one followed $50 \%$ of the time by one action and $50 \%$ of the time by two other actions equally and the other followed $50 \%$ of the time by one action and $50 \%$ by another. The low probability pairs consisted of one cue action followed by 3 actions with $25 \%, 12.5 \%$, and $12.5 \%$ probability, respectively. From the transitional probabilities we extracted two distinct measures: expectancy and predictability.

Expectancy was calculated as the negative conditional surprise (Hale, 2016; Hochberg \& Attneave, 1961; Weissbart et al., 2020) ascribed to the transition of the paired action that was most likely to occur, i.e., the most probable paired action, given that a particular action step happened (see Figure 2, dotted lines).

$$
-I\left(x_{\text {max }} \mid x_{t-1}\right)=-\log _{2} p\left(x_{\text {max }_{t}} \mid x_{t-1}\right)
$$

where $t$ indicates the current timepoint, $\mathrm{t}-1$ indicates the previous timepoint, $\mathrm{x}$ indicates any action, and xmax indicates the most likely action among all possible actions.

Shannon's surprise is often used to quantify how unexpected a past event is, by taking the negative logarithm of the probability of such an event. In this study, we adapted the formula to address our research question on predictive rather than reactive processes: instead of capturing the probability of the last event, it captures the probability of the most likely upcoming event, termed here expectancy.

In words, expectancy is the conditional surprise of the most likely action, which is computed as the negative logarithm of the probability of the action that is most likely to follow the previous action.

Predictability of action steps was dependent on the number of possible next action steps and their respective probabilities. Negative Conditional entropy $(\mathrm{H})$ (Collell \& Fauquet, 2015; Cover \& Thomas, n.d.; Hale, 2016; Hochberg \& Attneave, 1961) provides a measure that takes these aspects into account:

$$
-H\left(x_{t} \mid x_{t-1}\right)=\sum_{i} p\left(x_{t}^{i} \mid x_{t-1}\right) * \log _{2} p\left(x_{t}^{i} \mid x_{t-1}\right)
$$


where $\mathrm{i}$ indicates any action $\mathrm{x}$ included in $\mathrm{X}$, that is, the space of all possible actions. In words, conditional entropy refers to the entropy ascribed to an upcoming event when the prior event is taken into account. It is calculated as sum of the surprise of all possible events $x_{t}$ given that $x_{t-1}$ occurred, weighted by their probability of occurring (see Figure 2 , continuous lines).

The probabilities were selected to allow for a contrast between expectancy and predictability. More specifically, the selected probabilities enabled us to extract two levels of predictability for both high (75\%) and medium (50\%) probabilities: low predictability and high predictability. The medium probability - low predictability condition and the high probability - high predictability condition had virtually the same predictability while having different expectancy (see Figure 2, gray column). Thus, the design allowed for two cue actions to have the same expectancy but different predictability and two actions to have the same predictability but different expectancy. Since the experiment included three training videos before the EEG session (see below), the transitional probabilities were taken as established at the beginning of the EEG session.

\begin{tabular}{|c|c|c|c|c|c|c|c|}
\hline & Squeeze & Push & Tilt & Spin & Open & Slide & \\
\hline Cue $\quad \begin{array}{l}\text { Paired } \\
\end{array}$ & & $\square$ & 01 & 0 & I & $\therefore$ & $H$ \\
\hline & & 100 & 0 & 0 & 0 & 0 & 0.0 \\
\hline [ & 25 & & 12.5 & 25 & 12.5 & 25 & 2.2 \\
\hline & 25 & 0 & & 25 & 50 & 0 & 1.5 \\
\hline C & 0 & 0 & 12.5 & & 12.5 & 75 & 1.0 \\
\hline U & 50 & 0 & 0 & 50 & & 0 & 1.0 \\
\hline & 0 & 0 & 75 & & 25 & & 0.8 \\
\hline
\end{tabular}

Figure 2. Example of a transition matrix ruling the presented action sequences. Probability matrix describing the transitional probabilities between action pairs. Rows depict the first object-associated action of a transition (cue) and columns depict the second object of a pair (paired). e.g., Squeeze (first row) was always ( $p=100 \%$ ) followed by a Push (third column), whereas Open (fifth row) could be followed by Spin (fourth column, $p=50 \%$ ) or Squeeze (first column, $p=50 \%$ ). Expectancy is determined by the action with highest probability given the cue only, and shown in bold font on the figure. An example is highlighted in the figure: cells surrounded by dotted lines determine the expectancy after seeing Tilt (blue) or Slide (red). In contrast, Predictability depends on the probability weights of all the possible outcomes given the cue. For instance, cells surrounded by continuous lines determine the Predictability of prediction given Tilt (blue) or Slide (red). The grey column shows the levels of Predictability $(H)$ associated to each cue. e.g., Squeeze (first row) report $H=0$ since the is always $(p=100 \%)$ associated with push, Open(fifth row) report $\mathrm{H}=1$ since it could be followed by Spin (fourth column, $\mathrm{p}=50 \%$ ) or Squeeze (first column, $p=50 \%)$. Predictability levels in orange represent two different cues with virtually the same entropy level but with different expectancy. 


\subsection{Procedure}

\subsubsection{Training phase}

At the start of the training, participants collected a USB-drive and a booklet with instructions from the experimenter. Participants were instructed to watch three training videos at home, one video each day, during the three days preceding the test phase. The USB-drive contained a stand-alone program, implemented in Python (https://www.python.org) and made independent using the Pyinstaller library (https://www.pyinstaller.org). The software was created to automatically select the correct video for each session and to record feedback from the webcam while the training videos were presented. To avoid online data transfer and to lower the risk of any privacy violations the webcam videos were stored locally on the encrypted USB-dive. Participants were instructed to bring the USB-drive containing the recorded webcam videos back at the EEG testing day.

\subsubsection{Test phase: EEG session}

The EEG session took place on the day following the last training session. The EEG session consisted of an action observation phase followed by an action execution phase. Participants were informed about the procedure and informed consent was obtained. The experimenter fitted an EEG cap to the participant (ActiCap, Brain Products, Munich, Germany) with 32 active electrodes arranged according to the standard 10-20 configuration. Following capping, EEG was recorded continuously while participants sat in front of a computer screen in a shielded testing room. The EEG signal was referenced online to $\mathrm{FCz}$. During measurement, the signal was amplified using a BrainAmp amplifier, band-pass filtered $(0.1-125 \mathrm{~Hz})$, and digitized at $500 \mathrm{~Hz}$.

During the action observation phase, participants observed two videos with the same duration and statistical structure as the training videos. At the end of each video, participants could take a short break. Participants were shown the two test videos displayed on a monitor while sitting at approximately $60 \mathrm{~cm}$ distance from the screen. The video resulted in an image of $38 \times 28.5 \mathrm{~cm}$.

After participants had observed the two test videos, the action execution phase began. The purpose of this phase was to identify the sample-specific mu and beta frequency range, as defined by a decrease in power during overt movement relative to baseline (Meyer et al., 2016; Monroy et al., 2019). The experimenter placed the toy-box shown in the videos in front of the participant and provided them with instructions regarding the execution task: after being presented on the screen with an image of one of the six actions that could be performed on the toy stimulus, participants had to execute that action. To indicate the onset and offset of their action, participants were asked to press the spacebar of the keyboard in 
front of them. To familiarize participants with the task, two test trial preceded the action execution phase. Participants completed 30 trials.

\section{Data analysis}

\subsection{EEG processing}

The EEG data was analysed using Fieldtrip, an open source toolbox for Matlab (Oostenveld et al., 2011). Continuous data were segmented into 1s epochs as follows: Baseline epochs were extracted from the $1 \mathrm{~s}$ fixation cross period and the experimental epochs were analysed in the 1s time window of the still frame preceding the second action (i.e., the predictive time window, see Figure 1). Segments were de-trended, corrected by the mean of the entire segment. Epochs were visually inspected for major artifacts such as noisy channels or muscle artifacts. Independent component analysis (ICA) was run on the epochs to correct for eye blink components. Finally, epochs were again visually inspected for any remaining artifacts. After artifact rejection, epochs were transformed using a Fast Fourier transformation using the multitaper method (Hanning taper) to estimate power values in the sample-specific frequency ranges (see Identification of the mu and beta rhythm). Finally, power values across the central electrodes (C3, Cz, C4) were log-transformed and extracted for statistical analyses (Meyer et al., 2016; Monroy et al., 2019; Perry et al., 2010; Pineda \& Hecht, 2009). In addition, power values over occipital electrodes $(01, \mathrm{Oz}, \mathrm{O} 2)$ were log-transformed and extracted as comparison region (Meyer et al., 2016; Monroy et al., 2019; Perry et al., 2010).

\subsection{Identification of the mu and beta rhythm}

Continuous EEG data during the action execution was segmented in 1s epochs for data processing (see EEG processing). The epochs were selected in the central 1s window between the onset and offset of their action as indicated by participants pressing the spacebar. Power was analysed in the $2-30 \mathrm{~Hz}$ range over central electrodes $(C 3, C 4, C z)$ to visually identify the alpha and beta range in which action execution is expected by previous literature to elicit lower power than rest (baseline). Data inspection indicated a clear negative alpha peak around $8 \mathrm{~Hz}(+/-1 \mathrm{~Hz})$ and a less pronounced negative beta peak around $17(+/-1 \mathrm{~Hz})$ in which power during action execution was lower compared to baseline (the $500 \mathrm{~ms}$ before the onset of the action) (see Figure 3). The extracted sample-specific frequency ranges were used to compare our conditions of interest in the action observation phase. 

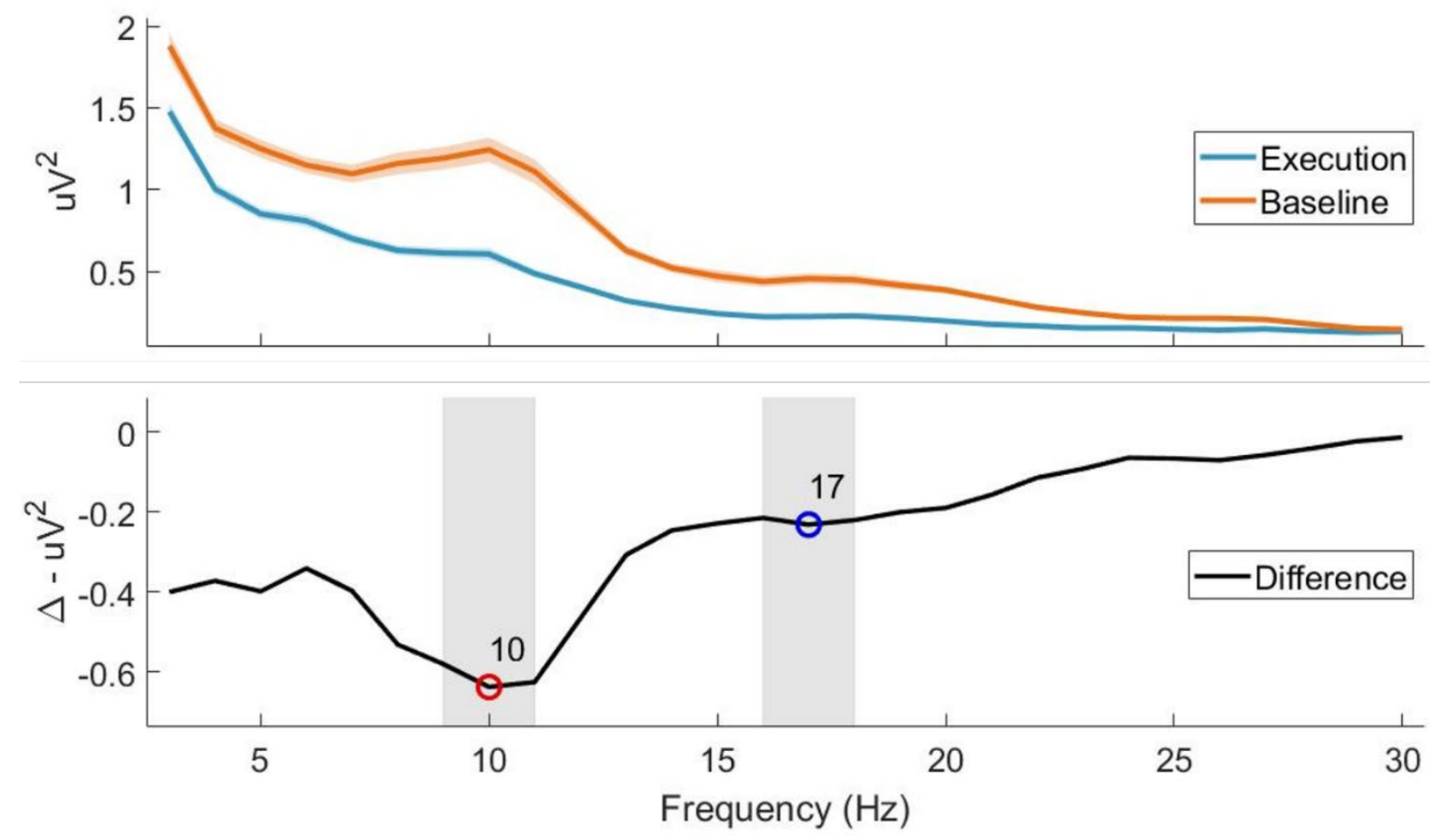

Figure 3. Power values as a function of frequency $(\mathrm{Hz})$ for the action execution and baseline trials. In the top plot the power spectra of the execution and the baseline (fixation cross) trials is shown. Shaded areas represent the standard error of the mean. In the bottom plot the difference between the execution and baseline power spectra is shown. This plot was used to visually identify the peaks of suppression during action execution. The mu rhythm peak is apparent between 9 and $11 \mathrm{~Hz}$. The beta rhythm is apparent between 16 and $18 \mathrm{~Hz}$.

\subsection{Statistical Analysis}

\subsubsection{Probability}

To test if sensorimotor areas were differently activated during the prediction of actions with different probabilities, we first applied a linear mixed-effect model (function 'Imer'; package 'Ime4' in R; (Bates et al., 2014)), fitted with restricted maximum likelihood. Subsequently, we extracted the F-test and p-values using the ANOVA function of the LmerTest package (Kuznetsova et al., 2017). Separate models were fitted with either mu or beta rhythm logpower over central sites $(C 3, C z, C 4)$ as the dependent variable. Condition $(100 \%, 75 \%, 50 \%$, $25 \%$, Baseline) was added as fixed within-subject factor. We expected suppression in these frequency bands in the channels over motor regions.

While suppression of mu power over central brain regions reflects motor processes, alpha power over occipital regions is related to visual processes (Bowman et al., 2017; Debnath et al., 2019; Fox et al., 2016; Hobson \& Bishop, 2016). To investigate the topographical specificity of the power reduction in the $9-11 \mathrm{~Hz}$ range the same linear mixedeffect model ANOVA was fitted using the mu rhythm log-power over the occipital channels $(01, \mathrm{Oz}, 01)$ as the dependent variable. Similarly to Monroy et al. (2019), we expected to find less suppression of alpha power during the baseline (fixation cross) in comparison to the 
predictive time window conditions (displaying the toy-box stimulus) regardless of the upcoming action probability.

\subsubsection{Interaction of expectancy and predictability}

Next, we investigated the interaction between expectancy and predictability in the prediction of the upcoming action. Separate linear mixed-effect model ANOVAs were fitted with mu and beta rhythm log-power over central sites $(C 3, C Z, C 4)$ as dependent variables. Expectancy (50\%, 75\%) and predictability (High, Low) were added as fixed within-subject factors. To account for repeated testing and the different distribution of activity over the sensorimotor area, we included a random intercept per electrode nested within participant (Ibrahim et al., 2013; Karas et al., 2019; Metzger et al., 2020; Verweij et al., 2014). All contrast analysis were tested for significance using Bonferroni corrections for multiple comparisons using the package Modelbased (Makowski et al., 2020).

\subsubsection{Mixed Models}

To determine on which information the participants based their prediction, we conducted multiple linear mixed-effect models fitted with maximum likelihood. Separate models were fitted with either mu or beta rhythm log-power over central sites (C3, Cz, C4) (Meyer et al., 2016; Monroy et al., 2019; Perry et al., 2010; Pineda \& Hecht, 2009) as the dependent variable. Expectancy and predictability levels were extracted for all the conditions and used as within-subject factor. For each frequency band three models were fitted: a Null model, the expectancy model, and the predictability model. The models were compared with each other and with the respective Null model using the Akaike's information criterion (AIC) (Akaike, 1974; Bozdogan, 1987; Vrieze, 2012). AIC determines the relative information value of the model using the maximum likelihood estimate and the number of parameters (independent variables) in the model. The smaller the AIC value, the better the model fit.

To account for repeated testing and the different distribution of activity over the areas, all the models included a random intercept per electrodes nested within participant (Ibrahim et al., 2013; Karas et al., 2019; Metzger et al., 2020; Verweij et al., 2014). All assumptions in terms of linearity, normality and homogeneity of residuals variance were verified and met for all models. 


\section{Results}

\subsection{Mu rhythm}

\subsubsection{Probability}

The linear mixed-effect model ANOVA revealed a significant effect of Probability $(F(4)=13.59$, $p<0.001$ ) over the sensorimotor cortex. Contrast analysis revealed that the mu rhythm was more suppressed when participants expected the appearance of an action compared to baseline, regardless of its level of probability (all $p<0.002$, see Figure 4 ). No differences were found between the different levels of probability. The mu rhythm activity over the sensorimotor cortex thus seems to reflect anticipatory motor activation during the prediction of an upcoming action regardless of its probability.

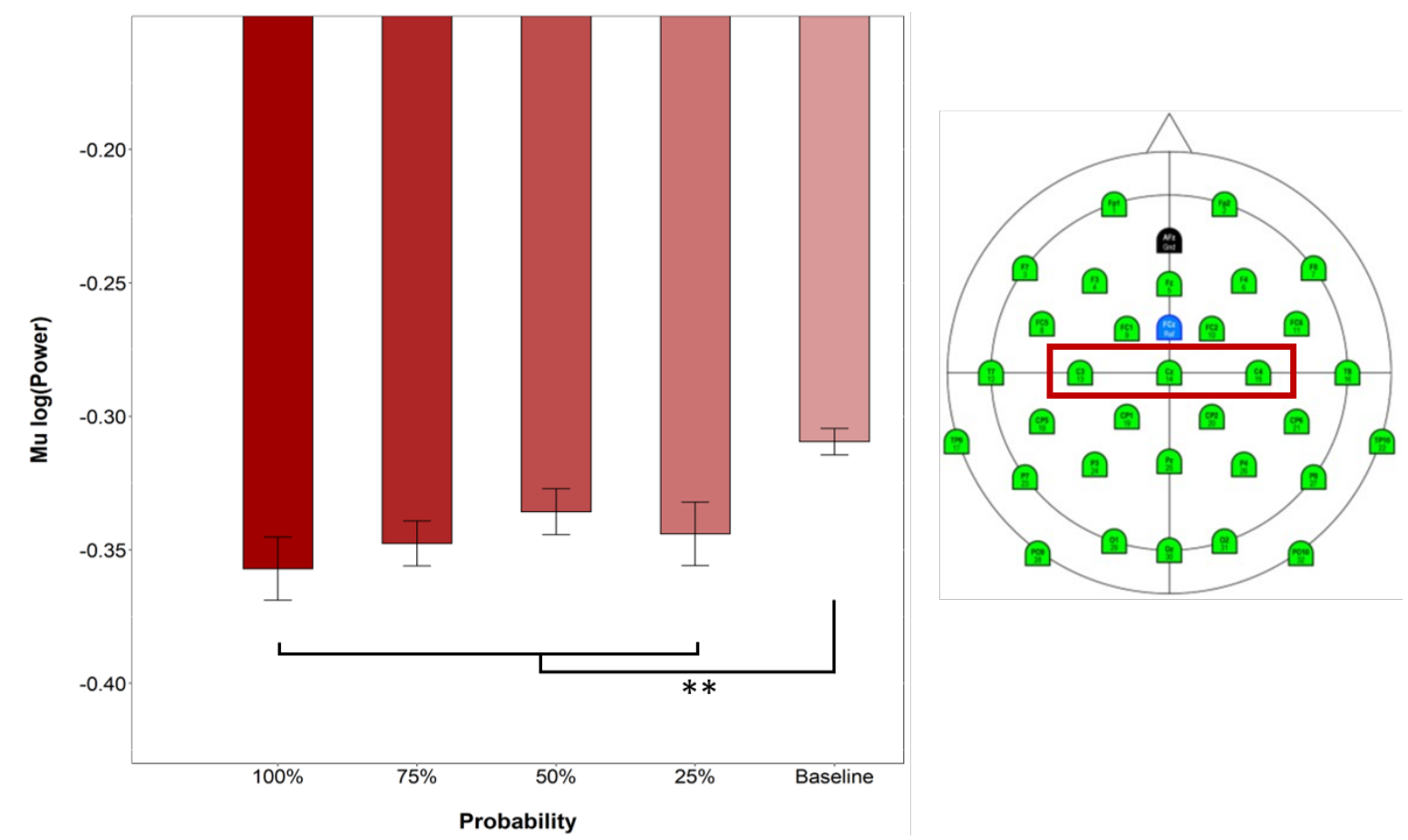

Figure 4. Linear mixed-effect model ANOVA. Log-transformed mu power over central channels overlaying motor regions (electrode layout depicted on the right; selected channels are marked in red). All probability levels were significantly different from baseline. Error bars represent the standard error of the mean. Please note that the $y$-axis does not start from 0 for visualisation purposes.

Contrary to our expectations, the linear mixed-effect model ANOVA conducted on the alpha frequency for electrodes over the occipital cortex revealed a significant effect of Probability $(F(4)=3.57, p=0.015)$. The contrast analysis comparison revealed significant differences between $25 \%$ and $75 \%$ as well as $25 \%$ and baseline (all $p<0.03$ ). When expecting an action with low probability (25\%), the occipital area was more active (i.e., power more suppressed) 
compared to when expecting actions with high probability (75\%) or no action (see Figure 5). The alpha rhythm is thought to reflect processing of visual stimuli and thus the observed reduction in occipital alpha power reflects enhanced visual attention in anticipation of a stimulus. On the basis of this data, we can therefore not exclude the possibility that observed power reduction in the 9-11 $\mathrm{Hz}$ range as observed over sensorimotor regions reflects a general suppression of the alpha rhythm, rather than a specific modulation of the sensorimotor mu rhythm.

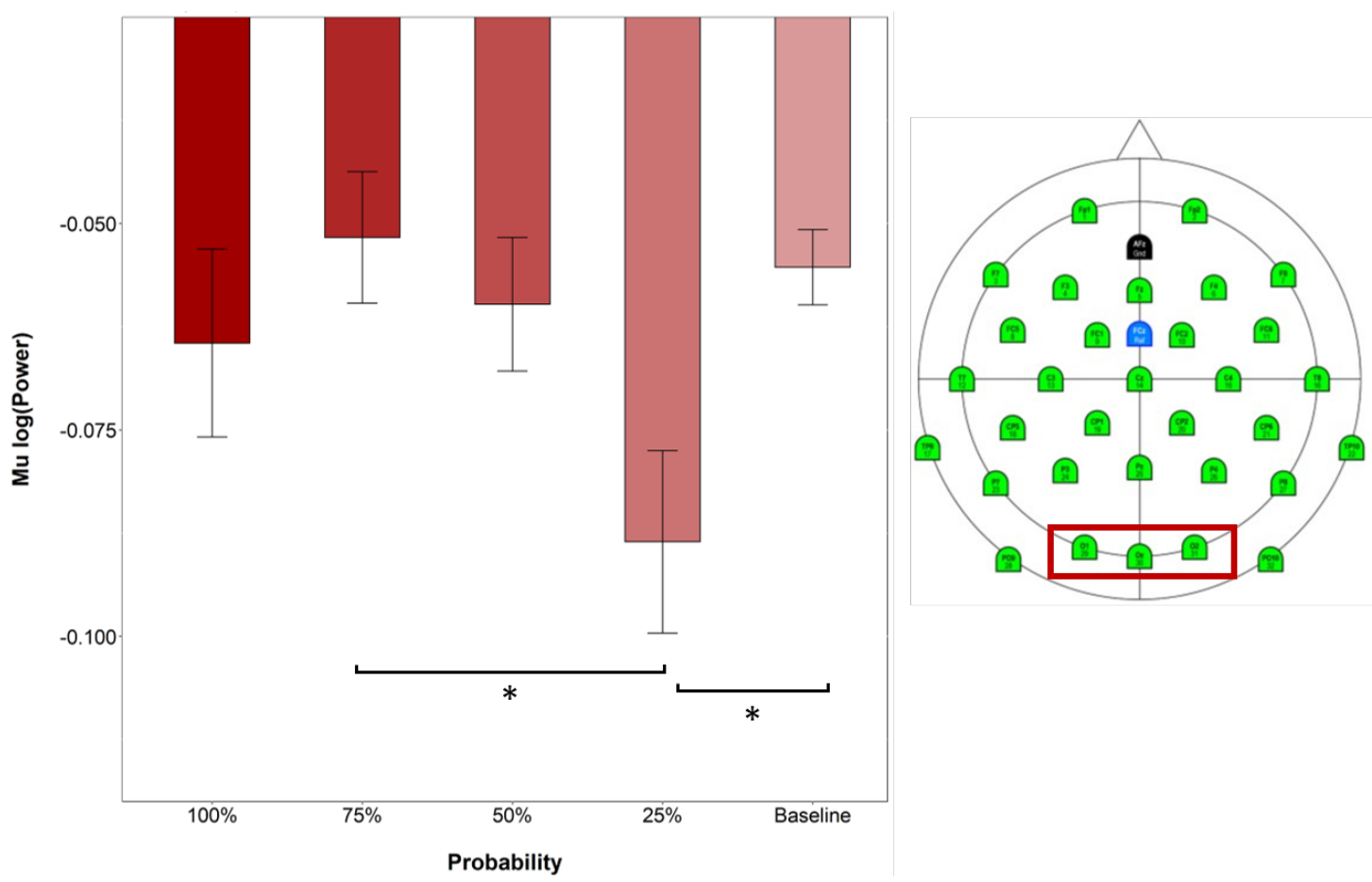

Figure 5. Linear mixed-effect model ANOVA. Log-transformed mu power over channels overlaying occipital regions (electrode layout depicted on the right; selected channels are outlined in red). $75 \%-25 \%$ and $25 \%$ - Baseline were significantly different. Error bars represent the standard error of the mean. Please note that the $y$-axis does not start from 0 for visualisation purposes.

\subsubsection{Interaction expectancy and predictability}

The ANOVA conducted to investigate the interaction between expectancy and predictability did not reveal any significant effects on mu power $(p=0.49)$. This is consistent with the previous analysis showing that the mu rhythm suppression was not modulated by the different levels of probability. 


\subsubsection{Mixed models}

Both expectancy and predictability models fitted on the mu rhythm power show no significant effect (expectancy: beta $=-6.75 e-03,95 \% \mathrm{Cl}[-4.56 e-03,0.02], t(14527)=$ 1.17, $p=0.242 ;$ Std. beta $=7.38 e-03 ;$ predictability: bet $a=$ $-5.55 e-03,95 \% \mathrm{Cl}$ [-5.10e-03, 0.02], $t(14527)=1.02$, $p=0.307$; Std. beta $=6.44 e-03)$. The Akaike information criterion was calculated for all the models and compared to the Null model (see Figure 6). Results show that the AIC of the expectancy and predictability models were higher than those for the Null model (Null: 18229.9; expectancy: 18230.53; predictability: 18230.86) indicating that neither expectancy nor predictability modulated the mu rhythm during the prediction of the upcoming action.

\subsection{Beta rhythm}

\subsubsection{Probability}

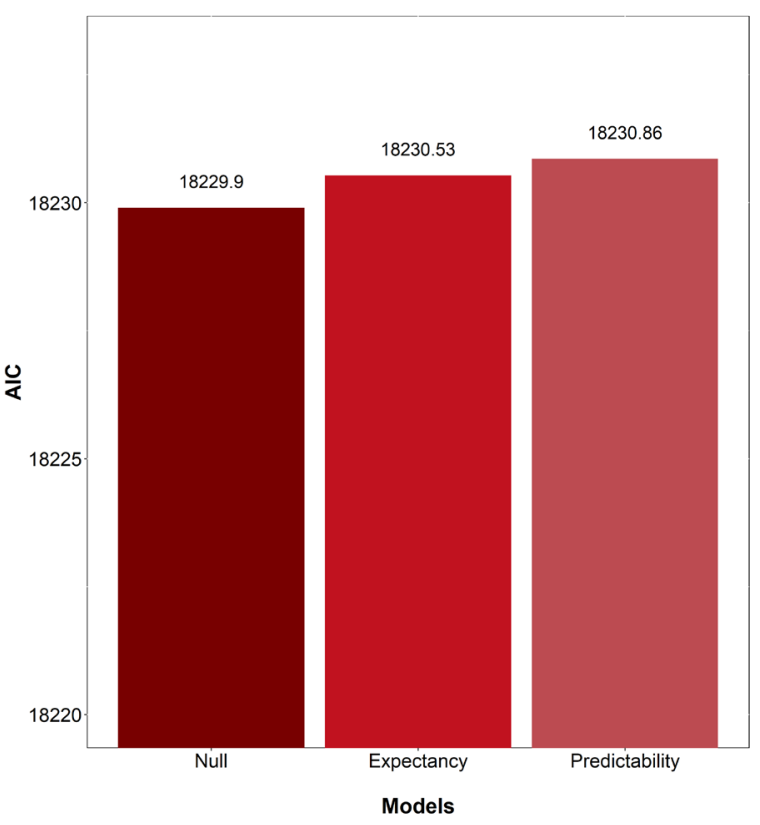

Figure 6. Model comparison mu rythm. AIC bar plot of the different models. AIC of the Null model was the lowest one indicating that expectancy and Predictability did not predict the mu rhythm activity over the motor area. Please note that the $y$-axis does not start from 0 for visualisation purposes.

The linear mixed-effect model ANOVA for beta power revealed a significant effect of Probability $(F(4)=18.78, p<0.001)$ over the sensorimotor cortex. Similarly to the results of the ANOVA with the dependent variable mu power, the contrast analysis test revealed that the beta rhythm was more suppressed when participants expected the appearance of an action compared to baseline (all $p<0.001$, see Figure 7 ). Additionally, the contrast analysis showed that the probabilities that differed from baseline also differed from the $25 \%$ probability level (all $p<0.024$ ). No significant difference was found between $25 \%$ probability and Baseline $(p=0.985)$. Thus, the beta rhythm seems to be sensitive to whether the probability of an upcoming action is very low compared to $50 \%$ or more. This sensitivity was not evident for the mu rhythm. 


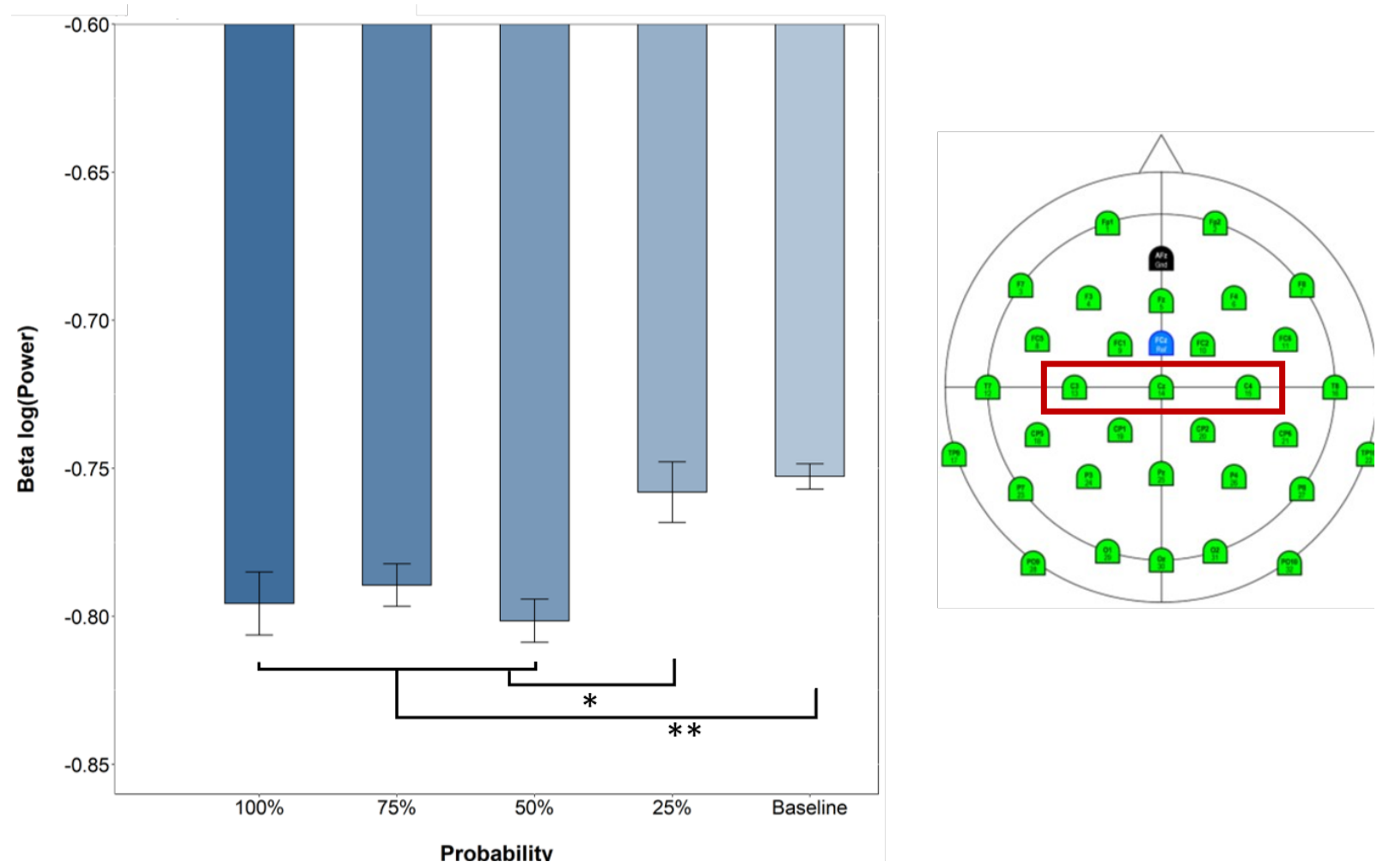

Figure 7. Linear mixed-effect model ANOVA. Log-transformed beta power over channels overlaying sensorimotor regions (electrode layout depicted on the right; selected channels are outlined in red). 100\%, $75 \%$ and $50 \%$ probability levels were significantly different from $25 \%$ and from baseline. Please note that the $y$-axis does not start from 0 for visualisation purposes.

\subsubsection{Interaction expectancy and predictability}

The linear mixed-effect model ANOVA exploring the relationship between expectancy and predictability revealed a significant interaction $(F(3)=7.93, p<0.001)$. Contrast analysis showed a significant difference between the level of predictability (High, Low) for the expectancy calculated over $50 \%(p=0.003)$ and $75 \%(p=0.014)$ probabilities (see Figure 8$)$. The contrast analysis revealed a significant difference between the expectancy calculated for $50 \%$ and $75 \%$ of probability in the Low predictability condition $(p<0.001)$ but not in the High condition $(p=0.174)$. These results show that there was an effect of predictability when predicting upcoming actions. While the effect of predictability on expectancy calculated for $75 \%$ is consistent with our hypothesis (i.e., more beta suppression for high compared to low predictability), the effect on expectancy calculated for $50 \%$ is in contrast with it (i.e., more beta suppression for low compared to high predictability). The effect of predictability on participants' anticipatory motor activity thus seems to be specific to different levels of expectancy. 


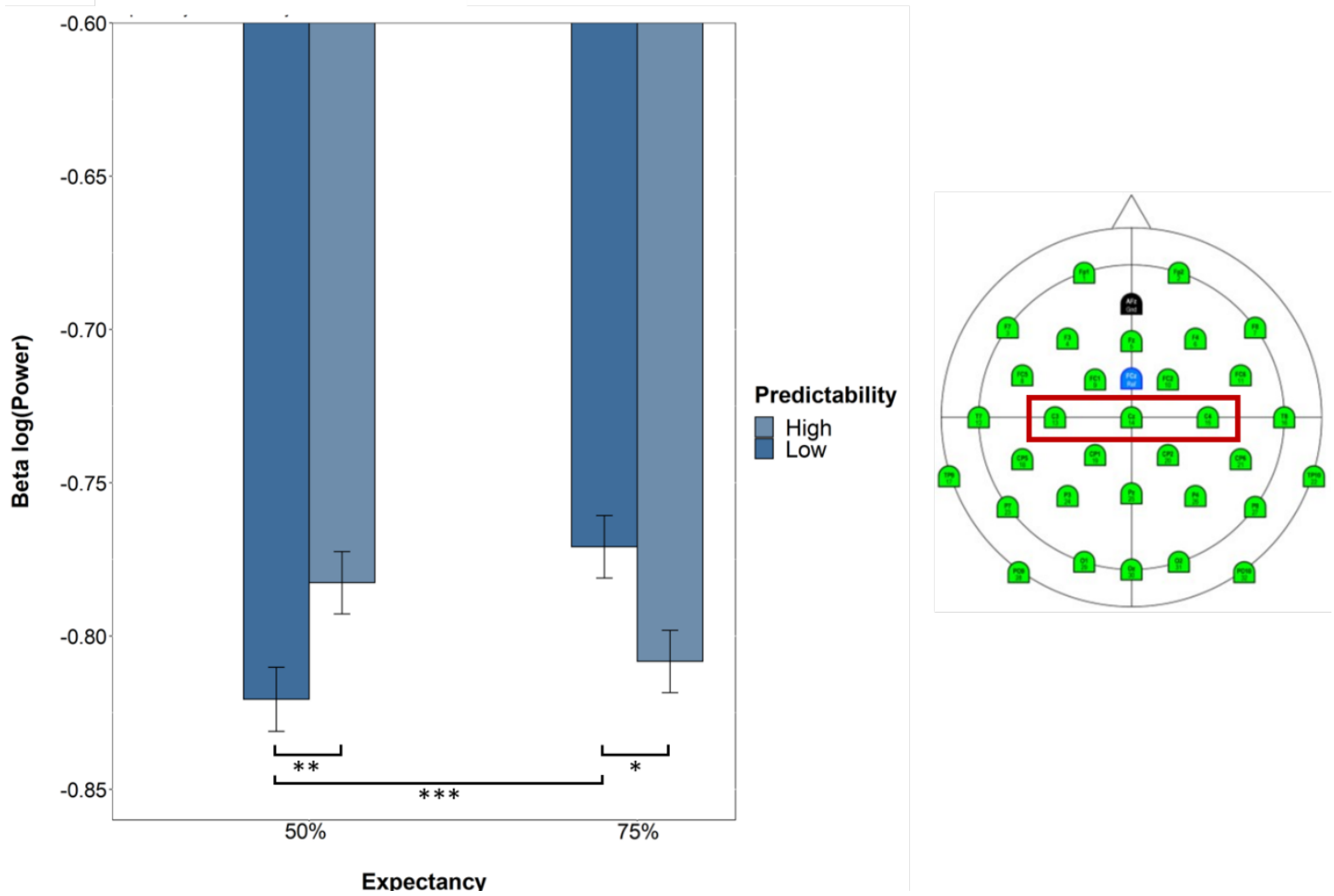

Figure 8. Linear mixed-effect model ANOVA. Anova performed to test the interaction between expectancy and predictability for log-transformed beta power over channels overlaying central regions (electrode layout depicted on the right; selected channels are outlined in red). There was a significant difference between the level of predictability (High, Low) for the expectancy calculated over $50 \%$ and $75 \%$ probabilities and a significant difference between the expectancy calculated for $50 \%$ and $75 \%$ of probability in the High predictability condition. Please note that the $y$-axis does not start from 0 for visualisation purposes.

\subsubsection{Mixed Models}

All the linear mixed-effect models fitted on the beta rhythm power showed a substantial explanatory power (all $R^{2}>2.6$ ). Both expectancy and predictability models showed a small positive effect with expectancy as beta $=-0.02, \mathrm{SE}=0.01,95 \% \mathrm{Cl}[0.00,0.03]$, std. beta $=0.02$ and predictability as beta $=-0.01, S E=0.01,95 \% \mathrm{Cl}[0.00,0.02]$, std. beta $=0.02$. The Akaike information criterion was calculated for all models. AIC of the expectancy and predictability models were smaller than those for the Null model (Null: 17430.45; expectancy: 17424.75; predictability: 17427.41) with expectancy being the best-fit model (see Figure 9). The results show that beta rhythm suppression better reflects the expectancy of the upcoming action and that participants were more engaged in predicting the most probable upcoming actions than the overall probability distribution (i.e. predictability).

\subsubsection{Exploratory Model}

Based on the interaction effect between expectancy and predictability on the beta rhythm we included an exploratory model in our mixed model comparison. The exploratory model 
was constructed to explore the hypothesis that participants predicted the most likely action but when multiple actions with the same probability were present, all these equiprobable actions were taken into account. This was the case for the low probability and for the medium probability with high predictability cues. In the former, the cue action was followed by three actions with respectively $25 \%$ probability and by two other actions with respectively $12.5 \%$ probability. In the latter, the cue action was followed by two paired actions with respectively $50 \%$ probability. To account for these equiprobable actions we extracted the conditional entropy for the paired actions that were most likely to follow the cue action.

$$
H\left(x_{\text {maxt }} \mid x_{t-1}\right)=\sum_{i=1}^{n x_{\text {max }_{t}}} p\left(x_{\text {maxt }}^{i} \mid x_{t-1}\right) * \log _{2} p\left(x_{\text {maxt }}^{i} \mid x_{t-1}\right)
$$

The model was fitted following the same procedure described in the Methods section. Similarly to the planned models, the exploratory model demonstrated substantial explanatory power $\left(R^{2}=0.2612\right)$. The model showed a significantly positive effect with beta $=-0.03,95 \%$ $\mathrm{Cl}[0.01,0.04], t(14527)=3.63, p<.001$; Std. beta $=0.03$. AIC of the exploratory model was extracted and compared to the other modes. AIC of the exploratory model was found to be the lowest with a score of 17419.249 (Null: 17430.45; expectancy: 17424.75; predictability: 17427.41; see Figure 9) demonstrating that the beta rhythm suppression over the motor area was best explained by the explorative model. This result shows that while participants were engaged in predicting the most probable upcoming action, they also consider the distribution of probabilities when predicting actions with the same likelihood. 


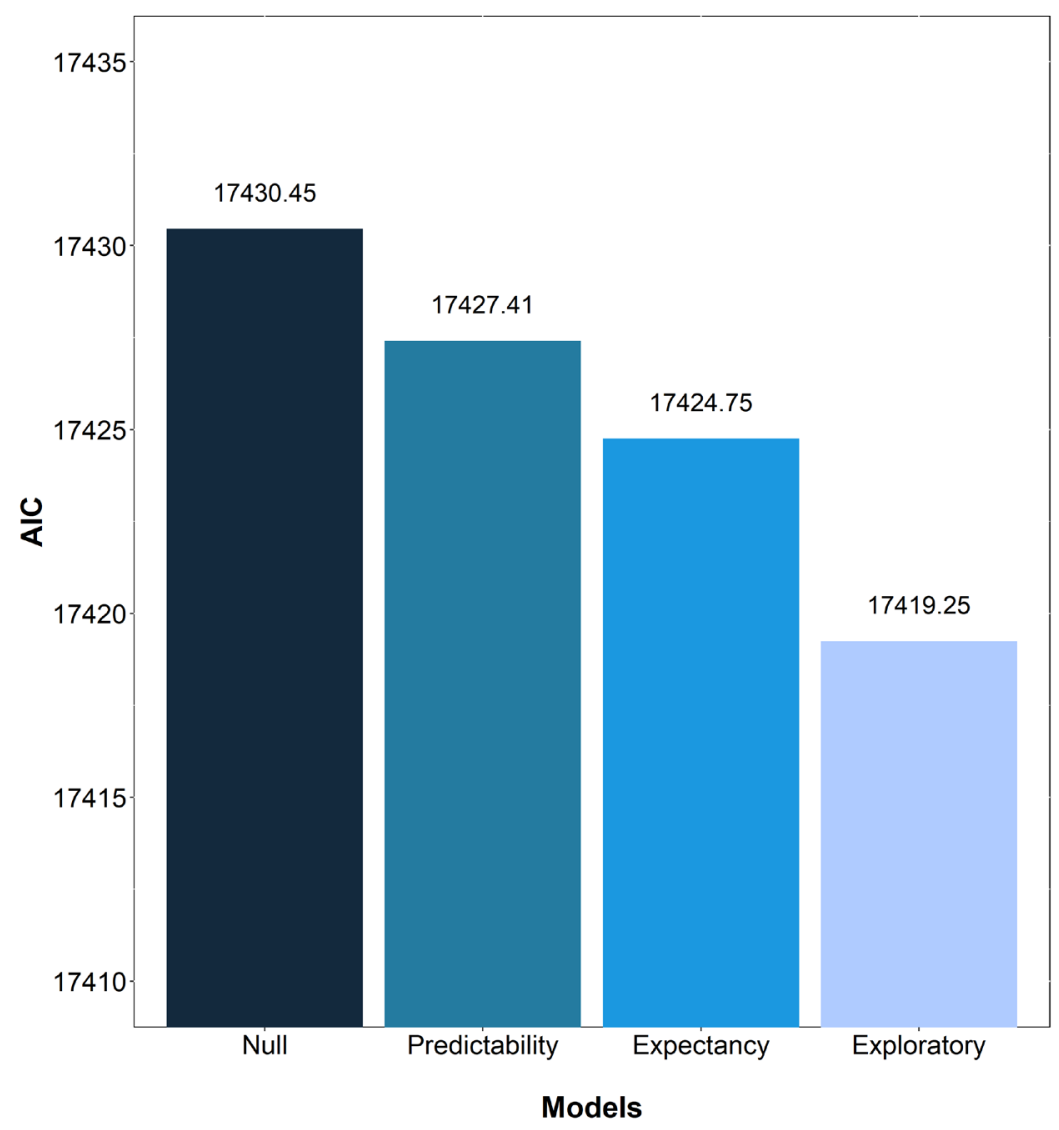

Figure 9. Model comparison beta rhythm. Barplot representing the AIC of the different models. AIC of the exploratory model was the lowest one of the planned models. The explorative model had the lowest AIC overall. Please note that the $y$-axis does not start from 0 for visualisation purposes. 


\section{Discussion}

Predicting actions is a fundamental ability that helps us comprehend what is happening in our environment and interact successfully with others. Making efficient and reliable predictions of others' actions thus plays an essential role in everyday life. Previous research on the role of the motor system in action prediction has primarily focused on simple goal-directed actions (Elsner et al., 2013; Kilner et al., 2007, 2004; Meyer et al., 2016; Monroy et al., 2019; Southgate et al., 2009). The present study systematically examined whether the activity of the motor system during action prediction is parametrically modulated by the probability of the upcoming actions. In particular, we investigated two distinct aspects of the statistical structure that reflect different prediction processes. Predictability, that reflects the prediction of the most likely action and expectancy that represent all possible actions and their relative probabilities. To explore which of these two processes underlie predictive motor activation in adults we extracted two statistical indices from the action sequences: expectancy and predictability. After a training phase where participants were exposed to the statistical structure, we measured the electrophysiological activity over the motor area focusing on the mu and beta frequencies. Decreased power in these frequency bands is associated with predictive motor activation.

\subsection{Mu rhythm: predicting actions independently of their probability}

We had expected a negative correlation between the probability of the upcoming actions and both power in mu and beta frequencies. Contrary to our expectations we did not find such a parametric modulation. We found differences between actions that had very low or zero probability and actions that had a high chance to occur, and no differences between the higher probability levels. In line with (Monroy et al. 2019), our results show that the mu rhythm was more suppressed when participants anticipated actions than when they were not, independently of their probability. In line with these results, the mu rhythm over the motor area showed no modulation in relation to the levels of expectancy or predictability. Together, our findings suggest that the mu rhythm serves a broader function of engagement during the anticipation of action and thus is enhanced when expecting an action, independently from its probability.

An alternative interpretation is that the suppression in the $9-11 \mathrm{~Hz}$ range reflects a suppression of the occipital alpha rhythm, rather than mu. The two frequency ranges overlap and it is possible that the general processing of visual stimuli, linked to the alpha range (Bazanova \& Vernon, 2014), could have influenced the power monitored during the predictive window. The analyses on the channels over the occipital area showed, contrary to what was expected, that the activity of the occipital area was modulated by the different levels of probability. In particular, the occipital area was more active when predicting low probability stimuli than when predicting stimuli with high probability or baseline. We hypothesize that in the low probability condition participants couldn't predict the action and its location on the 
toybox. Thus, they visually explored more the toybox resulting in a higher suppression of the alpha rhythm. While other studies dissociated mu and occipital alpha (e.g. (Debnath et al., 2019), we can on the basis of the current data not reliably exclude the possibility that the activity measured over the motor area was influenced by the alpha activity over occipital areas.

\subsection{Beta rhythm: predicting the most probable upcoming action}

The beta frequency was more suppressed when participants expected actions with high probability than when expecting actions with low probability or no action at all, but no parametric modulation was found. This result is in line with what was found by (Monroy et al. 2019). In this study, infant motor activity when predicting a deterministic action was different from baseline and the $25 \%$ level of probability. However, no difference was found between the $25 \%$ probability and baseline. We therefore assume that the level of probability was so low that the contingency was not detected and thus was not discernible from chance (or here the baseline). Interestingly the mixed models conducted on the beta frequency were significant, showing that both expectancy and predictability were modulating the betarhythm activity. We believe that this contrast in the results is due to subtle modulations of beta by the statistical structure. In fact, a more fine-grained analysis of how differences in beta relate to the different probabilistic features of the environment allowed us to detect effects that would not emerge when exploring the variables at a more superficial level (i.e., categorical differences between probabilities). When exploring whether expectancy or predictability underlie participants' action predictions as reflected in beta power modulations, we discovered that both expectancy and predictability showed increased explanatory power in comparison to the null model, with expectancy having the strongest explanatory power. This result shows that participants based their prediction on the probability of the specific, most likely action they expected to occur rather than all the different possibilities and their probability. However, the significant interaction between expectancy and predictability suggests that during action prediction multiple dimensions of our knowledge are taken into account to predict what will happen next.

In other words, while in the $75 \%$ condition we found that the activity of the motor system was higher when predictability was high, we found the opposite pattern in the $50 \%$ condition namely that the motor system was more active for low predictability. This might be due to how participants perceived the $50 \%$ high predictability condition. We believe that in this case participants had more difficulties in making a distinct prediction since both next actions had a $50 \%$ probability to appear. To examine this hypothesis, we analysed an exploratory model which was constructed to combine expectancy and predictability and their different strengths. For the conditions in which it was easy to identify the most probable action, the model focused on its expectancy without taking the other options into account. However, when several actions had the same highest the model took the number of those possible options into consideration. This model demonstrated the highest explanatory power of all 
models fit to the beta rhythm data, suggesting that participants flexibly adapted their prediction processes to the situation. Together these results indicate that depending on the content and difficulty in predicting which action will occur, different information is reflected in predictive motor activity. Future studies are needed to systematically test whether these two types of statistical information are indeed extracted in parallel and differently utilized depending on the situation.

\subsection{Action predictions in the motor system}

Our study focused on predictive activation of the motor system in anticipation of an upcoming action and explored whether and how neural oscillations are modulated by the likelihood of upcoming actions. Our results are in line with previous studies that demonstrate the role of the neural motor system in generating action predictions (Elsner et al., 2013; Kilner et al., 2007; Meyer et al., 2016; Monroy et al., 2019), and they support the hypothesis that the activity of motor areas is modulated by the statistical structure of the action stream (Braukmann et al., 2017; Monroy et al., 2019; Plata Bello et al., 2015; Rizzolatti et al., 1996; Rizzolatti \& Sinigaglia, 2010). Interestingly, we found a modulation of activity in the beta range by expectancy and predictability, while power in the mu range was independent of the statistical structure. The mu rhythm was attenuated when anticipating actions, but it did not show a modulation by either expectancy or predictability. Whereas both rhythms are associated with motor system activation (Angelini et al., 2018; Fox et al., 2016; Hobson \& Bishop, 2017; McFarland et al., 2000; Muthukumaraswamy et al., 2004), some studies suggest that the mu and beta rhythms have different origins in the brain (Ritter et al., 2009) and have a different functionality (Brinkman et al., 2014; Stolk et al., 2019). In line with previous findings, our results suggest that while the beta frequency is sensitive to how probable an action is (van Pelt et al., 2016), the mu rhythm may reflect a general mechanism of activation in the motor system that reflects the anticipation of an action independently by its probability (Braukmann et al., 2017).

While this study explored the activity of the motor system during action prediction and tried to distill which statistical information this predictive activity is reflecting, it did not address the role of the motor area for the generation of the prediction per se. Is the motor system generating action predictions or are the predictions generated elsewhere and the motor area rather relaying this information? Recent studies suggest that the motor system receives an integrated signal from the sensory cortices and deeper structures. This signal is then processed into an action prediction by the motor area that creates a representation of the action that is about to occur (Ahlheim et al., 2014; Bornstein \& Daw, 2012; Harrison et al., 2006; Kahl \& Kopp, 2018; Monroy et al., 2018, 2019; Theves et al., 2021). However, since the present study was not designed to answer this question, future studies are needed to answer exactly what role the motor area plays in the generation of action predictions. 
Furthermore, this study investigated the activity of sensorimotor areas during action prediction, focusing on central channels $(\mathrm{C} 3, \mathrm{Cz}, \mathrm{C} 4)$. Given the relatively low spatial accuracy offered by a 32-channel EEG system, we are aware that the signal recorded in this study most likely reflects activation from a broader set of areas (including pre-motor, primary motor, and primary sensory cortices). We selected central channels to pick up activity of the sensorimotor cortices with as little interference from non-target areas as possible. To what extent other brain areas process regularities in observed actions and utilize them to anticipate upcoming actions remains an open question. Given the relatively low spatial accuracy, we believe that future studies using methodologies with higher resolution, such as MEG, $\mathrm{FMRI}$, or fNIRS, are needed to examine spatially distinct activity patterns over the brain related to the prediction of actions and their statistical structure.

In conclusion, the present EEG study shows that human adults can learn statistical information in sequences of actions they observe and use this knowledge to generate action predictions in their neural motor system. In accordance with recent empirical work suggesting distinct functional roles for the $\mathrm{mu}$ and beta rhythms and linking beta-oscillations to predictions in the motor system (Monroy et al., 2019; Ondobaka et al., 2015; Palmer et al., $2019,2016)$, we found that the beta rhythm is modulated by the statistical structure of the stimuli while the mu rhythm reflects a general activation of the motor system during the anticipation of an action. 


\section{Data availability}

Anonymised data can be found via the following

URL: https://data.donders.ru.nl/collections/di/dcc/DSC 2019.00043007

\section{Code availability}

The Matalb and $\mathrm{R}$ code used to analyze the data can be found on the github repository:

https://github.com/TommasoGhilardi/Predictive-motor-activation

\section{Funding}

This project was funded by the European Union's Horizon 2020 Marie Skłodowska-Curie ITN project MOTION (grant agreement $n^{\circ}$ 765298).

\section{Competing interests}

The authors have no affiliation with any organization with a direct or indirect financial interest in the subject matter discussed in the manuscript. 


\section{References}

Ahlheim, C., Stadler, W., \& Schubotz, R. I. (2014). Dissociating dynamic probability and predictability in observed actions an fMRI study. Frontiers in Human Neuroscience, 8(May), 1-13. https://doi.org/10.3389/fnhum.2014.00273

Akaike, H. (1974). A new look at the statistical model identification. IEEE Transactions on Automatic Control, 19(6), 716-723. https://doi.org/10.1109/tac.1974.1100705

Angelini, M., Fabbri-Destro, M., Lopomo, N. F., Gobbo, M., Rizzolatti, G., \& Avanzini, P. (2018). Perspective-dependent reactivity of sensorimotor mu rhythm in alpha and beta ranges during action observation: an EEG study. Scientific Reports, 8(1), 12429. https://doi.org/10.1038/s41598-018-30912-w

Bach, P., Nicholson, T., \& Hudson, M. (2014). The affordance-matching hypothesis: how objects guide action understanding and prediction. Frontiers in Human Neuroscience, 8, 254. https://doi.org/10.3389/fnhum.2014.00254

Baldwin, D., Andersson, A., Saffran, J., \& Meyer, M. (2008). Segmenting dynamic human action via statistical structure. Cognition, 106(3), 1382-1407. https://doi.org/10.1016/j.cognition.2007.07.005

Bates, D., Mächler, M., Bolker, B., \& Walker, S. (2014). Fitting Linear Mixed-Effects Models using Ime4. In arXiv [stat.CO] (Issue 1, pp. 1-48). arXiv. https://doi.org/10.18637/jss.v067.i01

Bazanova, O. M., \& Vernon, D. (2014). Interpreting EEG alpha activity. Neuroscience and Biobehavioral Reviews, 44, 94-110. https://doi.org/10.1016/j.neubiorev.2013.05.007

Bornstein, A. M., \& Daw, N. D. (2012). Dissociating hippocampal and striatal contributions to sequential prediction learning. The European Journal of Neuroscience, 35(7), 10111023. https://doi.org/10.1111/j.1460-9568.2011.07920.x

Bowman, L. C., Bakermans-Kranenburg, M. J., Yoo, K. H., Cannon, E. N., Vanderwert, R. E., Ferrari, P. F., van IJzendoorn, M. H., \& Fox, N. A. (2017). The mu-rhythm can mirror: Insights from experimental design, and looking past the controversy. Cortex; a Journal Devoted to the Study of the Nervous System and Behavior, 96, 121-125. https://doi.org/10.1016/j.cortex.2017.03.025

Bozdogan, H. (1987). Model selection and Akaike's Information Criterion (AIC): The general theory and its analytical extensions. Psychometrika, 52(3), 345-370. https://doi.org/10.1007/bf02294361

Braukmann, R., Bekkering, H., Hidding, M., Poljac, E., Buitelaar, J. K., \& Hunnius, S. (2017). Predictability of action sub-steps modulates motor system activation during the observation of goal-directed actions. Neuropsychologia, 103(February), 44-53. https://doi.org/10.1016/j.neuropsychologia.2017.07.009

Brinkman, L., Stolk, A., Dijkerman, H. C., de Lange, F. P., \& Toni, I. (2014). Distinct Roles for Alpha- and Beta-Band Oscillations during Mental Simulation of Goal-Directed Actions. Journal of Neuroscience, 34(44), 14783-14792. https://doi.org/10.1523/JNEUROSCI.2039-14.2014

Caetano, G., Jousmä, V., \& Hari, R. (2007). Actor's and observer's primary motor cortices stabilize similarly after seen or heard motor actions.

Collell, G., \& Fauquet, J. (2015). Brain activity and cognition: a connection from thermodynamics and information theory. Frontiers in Psychology, 6, 818. https://doi.org/10.3389/fpsyg.2015.00818 
Cover, \& Thomas. (n.d.). Entropy, relative entropy and mutual information. Elements of Information

Theory.

http://www.cs.columbia.edu/ vh/courses/LexicalSemantics/Association/Cover\&Tho mas-Ch2.pdf

Debnath, R., Salo, V. C., Buzzell, G. A., Yoo, K. H., \& Fox, N. A. (2019). Mu rhythm desynchronization is specific to action execution and observation: Evidence from timefrequency and connectivity analysis. Neurolmage, 184(June 2018), 496-507. https://doi.org/10.1016/j.neuroimage.2018.09.053

Elsner, C., D'Ausilio, A., Gredebäck, G., Falck-Ytter, T., \& Fadiga, L. (2013). The motor cortex is causally related to predictive eye movements during action observation. Neuropsychologia, 51(3), 488-492. https://doi.org/10.1016/j.neuropsychologia.2012.12.007

Fiser, J., \& Aslin, R. N. (2002). Statistical Learning of Higher-Order Temporal Structure from Visual Shape Sequences. Journal of Experimental Psychology: Learning Memory and Cognition, 28(3), 458-467. https://doi.org/10.1037/0278-7393.28.3.458

Flanagan, J. R., \& Johansson, R. S. (2003). Action plans used in action observation. Nature, 424(6950), 769-771. https://doi.org/10.1038/nature01861

Fox, N. A., Yoo, K. H., Bowman, L. C., Cannon, E. N., Ferrari, P. F., Bakermans-Kranenburg, M. J., Vanderwert, R. E., \& Van IJzendoorn, M. H. (2016). Assessing human mirror activity With EEG mu rhythm: A meta-analysis. Psychological Bulletin, 142(3), 291-313. https://doi.org/10.1037/bul0000031

Hale, J. (2016). Information-theoretical complexity metrics. Language and Linguistics Compass, 10(9), 397-412. https://doi.org/10.1111/Inc3.12196

Harrison, L. M., Duggins, A., \& Friston, K. J. (2006). Encoding uncertainty in the hippocampus. Neural Networks: The Official Journal of the International Neural Network Society, 19(5), 535-546. https://doi.org/10.1016/j.neunet.2005.11.002

Hobson, H. M., \& Bishop, D. V. M. (2016). Mu suppression - A good measure of the human mirror neuron system? Cortex; a Journal Devoted to the Study of the Nervous System and Behavior, 82, 290-310. https://doi.org/10.1016/j.cortex.2016.03.019

Hobson, H. M., \& Bishop, D. V. M. (2017). The interpretation of mu suppression as an index of mirror neuron activity: past, present and future. Royal Society Open Science, 4(3), 160662. https://doi.org/10.1098/rsos.160662

Hochberg, J., \& Attneave, F. (1961). Applications of Information Theory to Psychology: A Summary of Basic Concepts, Methods, and Results. In The American Journal of Psychology (Vol. 74, Issue 2, p. 319). https://doi.org/10.2307/1419430

Hudson, M., Nicholson, T., Simpson, W. A., Ellis, R., \& Bach, P. (2016). One step ahead: The perceived kinematics of others' actions are biased toward expected goals. Journal of Experimental Psychology. General, 145(1), 1-7. https://doi.org/10.1037/xge0000126

Ibrahim, G. M., Anderson, R., Akiyama, T., Ochi, A., Otsubo, H., Singh-Cadieux, G., Donner, E., Rutka, J. T., Snead, O. C., 3rd, \& Doesburg, S. M. (2013). Neocortical pathological highfrequency oscillations are associated with frequency-dependent alterations in functional network topology. Journal of Neurophysiology, 110(10), 2475-2483. https://doi.org/10.1152/jn.00034.2013

Kahl, S., \& Kopp, S. (2018). A Predictive Processing Model of Perception and Action for SelfOther Distinction. Frontiers in Psychology, 9(DEC). https://doi.org/10.3389/fpsyg.2018.02421 
Karas, P. J., Magnotti, J. F., Metzger, B. A., Zhu, L. L., Smith, K. B., Yoshor, D., \& Beauchamp, M. S. (2019). The visual speech head start improves perception and reduces superior temporal cortex responses to auditory speech. ELife, 8. https://doi.org/10.7554/eLife.48116

Kilner, J. M. (2011). More than one pathway to action understanding. Trends in Cognitive Sciences, 15(8), 352-357. https://doi.org/10.1016/j.tics.2011.06.005

Kilner, J. M., Friston, K. J., \& Frith, C. D. (2007). Predictive coding: An account of the mirror neuron system. Cognitive Processing, 8(3), 159-166. https://doi.org/10.1007/s10339007-0170-2

Kilner, J. M., Vargas, C., Duval, S., Blakemore, S.-J., \& Sirigu, A. (2004). Motor activation prior to observation of a predicted movement. Nature Neuroscience, 7(12), 1299-1301. https://doi.org/10.1038/nn1355

Kuznetsova, A., Brockhoff, P. B., \& Christensen, R. H. B. (2017). ImerTest Package: Tests in Linear Mixed Effects Models. Journal of Statistical Software, 82(13). https://doi.org/10.18637/jss.v082.i13

Makowski, D., Lüdecke, D., \& Ben-Shachar, M. S. (2020). Modelbased: Estimation of modelbased predictions, contrasts and means. CRAN. https://github. com/easys tats/model based.

McFarland, D. J., Miner, L. A., Vaughan, T. M., \& Wolpaw, J. R. (2000). Mu and beta rhythm topographies during motor imagery and actual movements. Brain Topography, 12(3), 177-186. https://doi.org/10.1023/A:1023437823106

Metzger, B. A., Magnotti, J. F., Wang, Z., Nesbitt, E., Karas, P. J., Yoshor, D., \& Beauchamp, M. S. (2020). Responses to visual speech in human posterior superior temporal gyrus examined with iEEG deconvolution. The Journal of Neuroscience: The Official Journal of the Society for Neuroscience, 40(36), 6938-6948. https://doi.org/10.1523/JNEUROSCI.0279-20.2020

Meyer, M., Braukmann, R., Stapel, J. C., Bekkering, H., \& Hunnius, S. (2016). Monitoring others' errors: The role of the motor system in early childhood and adulthood. The British Journal of Developmental Psychology, 34(1), 66-85. https://doi.org/10.1111/bjdp.12101

Monroy, C. D., Gerson, S. A., \& Hunnius, S. (2018). Translating visual information into action predictions: Statistical learning in action and nonaction contexts. Memory \& Cognition, 46(4), 600-613. https://doi.org/10.3758/s13421-018-0788-6

Monroy, C. D., Meyer, M., Schröer, L., Gerson, S. A., \& Hunnius, S. (2019). The infant motor system predicts actions based on visual statistical learning. Neurolmage, 185(December), 947-954. https://doi.org/10.1016/j.neuroimage.2017.12.016

Muthukumaraswamy, S. D., Johnson, B. W., Gaetz, W. C., \& Cheyne, D. O. (2006). Neural processing of observed oro-facial movements reflects multiple action encoding strategies in the human brain. Brain Research, 1071(1), 105-112. https://doi.org/10.1016/j.brainres.2005.11.053

Muthukumaraswamy, S. D., Johnson, B. W., \& McNair, N. A. (2004). Mu rhythm modulation during observation of an object-directed grasp. Cognitive Brain Research, 19(2), 195201. https://doi.org/10.1016/j.cogbrainres.2003.12.001

Oberman, L. M., Hubbard, E. M., McCleery, J. P., Altschuler, E. L., Ramachandran, V. S., \& Pineda, J. A. (2005). EEG evidence for mirror neuron dysfunction in autism spectrum disorders. Cognitive Brain Research, 24(2), 190-198. https://doi.org/10.1016/j.cogbrainres.2005.01.014 
Ondobaka, S., De Lange, F. P., Wittmann, M., Frith, C. D., \& Bekkering, H. (2015). Interplay between conceptual expectations and movement predictions underlies action understanding. Cerebral Cortex , 25(9), 2566-2573. https://doi.org/10.1093/cercor/bhu056

Oostenveld, R., Fries, P., Maris, E., \& Schoffelen, J.-M. (2011). FieldTrip : Open Source Software for Advanced Analysis of MEG , EEG , and Invasive Electrophysiological Data. 2011. https://doi.org/10.1155/2011/156869

Palmer, C. E., Auksztulewicz, R., Ondobaka, S., \& Kilner, J. M. (2019). Sensorimotor beta power reflects the precision-weighting afforded to sensory prediction errors. Neurolmage, 200(February), 59-71. https://doi.org/10.1016/j.neuroimage.2019.06.034

Palmer, C. E., Bunday, K. L., Davare, M., \& James, M. K. (2016). A Causal Role for Primary Motor Cortex in Perception of Observed Actions. Journal of Cognitive Neuroscience, 24(6), 1275-1285. https://doi.org/10.1162/jocn

Perry, A., Troje, N. F., \& Bentin, S. (2010). Exploring motor system contributions to the perception of social information: Evidence from EEG activity in the mu/alpha frequency range. Social Neuroscience, 5(3), 272-284. https://doi.org/10.1080/17470910903395767

Pineda, J. A., \& Hecht, E. (2009). Mirroring and mu rhythm involvement in social cognition: are there dissociable subcomponents of theory of mind? Biological Psychology, 80(3), 306-314. https://doi.org/10.1016/j.biopsycho.2008.11.003

Plata Bello, J., Modroño, C., Marcano, F., \& González-Mora, J. L. (2015). Modulation in the mirror neuron system when action prediction is not satisfied. The European Journal of Neuroscience, 41(7), 940-948. https://doi.org/10.1111/ejn.12850

Ritter, P., Moosmann, M., \& Villringer, A. (2009). Rolandic alpha and beta EEG rhythms' strengths are inversely related to fMRI-BOLD signal in primary somatosensory and motor cortex. Human Brain Mapping, 30(4), 1168-1187. https://doi.org/10.1002/hbm.20585

Rizzolatti, G., Fadiga, L., Gallese, V., \& Fogassi, L. (1996). Premotor cortex and the recognition of motor actions. Cognitive Brain Research, 3(2), 131-141. https://doi.org/10.1016/0926-6410(95)00038-0

Rizzolatti, G., \& Sinigaglia, C. (2010). The functional role of the parieto-frontal mirror circuit: interpretations and misinterpretations. Nature Reviews Neuroscience, 11(4), 264-274. https://doi.org/10.1038/nrn2805

Rutkowska, J. M., Meyer, M., \& Hunnius, S. (2021). Adults do not distinguish action intentions based on movement kinematics presented in naturalistic settings. Brain Sciences, 11(6), 821. https://doi.org/10.3390/brainsci11060821

Southgate, V., Johnson, M. H., Osborne, T., \& Csibra, G. (2009). Predictive motor activation during action observation in human infants. Biology Letters, 5(6), 769-772. https://doi.org/10.1098/rsbl.2009.0474

Stadler, W., Springer, A., Parkinson, J., \& Prinz, W. (2012). Movement kinematics affect action prediction: comparing human to non-human point-light actions. Psychological Research, 76(4), 395-406. https://doi.org/10.1007/s00426-012-0431-2

Stapel, J. C., Hunnius, S., \& Bekkering, H. (2012). Online prediction of others' actions: the contribution of the target object, action context and movement kinematics. Psychological Research, 76(4), 434-445. https://doi.org/10.1007/s00426-012-0423-2 
Stapel, J. C., Hunnius, S., Meyer, M., \& Bekkering, H. (2016). Motor system contribution to action prediction: Temporal accuracy depends on motor experience. Cognition, 148, 71-78. https://doi.org/10.1016/j.cognition.2015.12.007

Stapel, J. C., Hunnius, S., van Elk, M., \& Bekkering, H. (2010). Motor activation during observation of unusual versus ordinary actions in infancy. Social Neuroscience, 5(5), 451-460. https://doi.org/10.1080/17470919.2010.490667

Stolk, A., Brinkman, L., Vansteensel, M. J., Aarnoutse, E., Leijten, F. S. S., Dijkerman, C. H., Knight, R. T., de Lange, F. P., \& Toni, I. (2019). Electrocorticographic dissociation of alpha and beta rhythmic activity in the human sensorimotor system. ELife, 8, 1-24. https://doi.org/10.7554/eLife.48065

Theves, S., Neville, D. A., Fernández, G., \& Doeller, C. F. (2021). Learning and Representation of Hierarchical Concepts in Hippocampus and Prefrontal Cortex. The Journal of Neuroscience: The Official Journal of the Society for Neuroscience, 41(36), 7675-7686. https://doi.org/10.1523/JNEUROSCI.0657-21.2021

Tzagarakis, C., Ince, N. F., Leuthold, A. C., \& Pellizzer, G. (2010). Beta-band activity during motor planning reflects response uncertainty. The Journal of Neuroscience: The Official Journal of the Society for Neuroscience, 30(34), 11270-11277. https://doi.org/10.1523/JNEUROSCI.6026-09.2010

van Pelt, S., Heil, L., Kwisthout, J., Ondobaka, S., van Rooij, I., \& Bekkering, H. (2016). Betaand gamma-band activity reflect predictive coding in the processing of causal events. Social Cognitive and Affective Neuroscience, 11(6), 973-980. https://doi.org/10.1093/scan/nsw017

Verweij, I. M., Romeijn, N., Smit, D. J., Piantoni, G., Van Someren, E. J., \& van der Werf, Y. D. (2014). Sleep deprivation leads to a loss of functional connectivity in frontal brain regions. BMC Neuroscience, 15(1), 88. https://doi.org/10.1186/1471-2202-15-88

Vrieze, S. I. (2012). Model selection and psychological theory: a discussion of the differences between the Akaike information criterion (AIC) and the Bayesian information criterion (BIC). Psychological Methods, 17(2), 228-243. https://doi.org/10.1037/a0027127

Weissbart, H., Kandylaki, K. D., \& Reichenbach, T. (2020). Cortical Tracking of Surprisal during Continuous Speech Comprehension. Journal of Cognitive Neuroscience, 32(1), 155166. https://doi.org/10.1162/jocn_a_01467

Wolpert, D. M., Doya, K., \& Kawato, M. (2003). A unifying computational framework for motor control and social interaction. Philosophical Transactions of the Royal Society of London. Series B, Biological Sciences, 358(1431), 593-602. https://doi.org/10.1098/rstb.2002.1238 